\title{
On the 5G Communications: Fractal-Shaped Antennas for PPDR Applications
}

\author{
Mihai-Virgil Nichita $\left(\mathbb{D},{ }^{1}\right.$ Maria-Alexandra Paun $\mathbb{D}^{2},{ }^{2}$ Vladimir-Alexandru Paun $\left(\mathbb{D},{ }^{3}\right.$ \\ and Viorel-Puiu Paun (iD) \\ ${ }^{1}$ Doctoral School, Faculty of Applied Sciences, University Politehnica of Bucharest, 313 Splaiul Independentei, Sector 6, \\ Bucharest RO-060042, Romania \\ ${ }^{2}$ School of Engineering, Swiss Federal Institute of Technology (EPFL), Route Cantonale, Lausanne 1015, Switzerland \\ ${ }^{3}$ Five Rescue Research Laboratory, 35 Quai d'Anjou, Paris 75004, France \\ ${ }^{4}$ Physics Department, Faculty of Applied Sciences, University Politehnica of Bucharest, 313 Splaiul Independentei, Sector 6, \\ Bucharest RO-060042, Romania \\ ${ }^{5}$ Academy of Romanian Scientists, 54 Splaiul Independentei, Sector 5, Bucharest RO-050094, Romania
}

Correspondence should be addressed to Maria-Alexandra Paun; maria_paun2003@yahoo.com

Received 13 July 2020; Revised 17 October 2020; Accepted 1 June 2021; Published 19 June 2021

Academic Editor: Átila Bueno

Copyright (C) 2021 Mihai-Virgil Nichita et al. This is an open access article distributed under the Creative Commons Attribution License, which permits unrestricted use, distribution, and reproduction in any medium, provided the original work is properly cited.

\begin{abstract}
In this study, one method of using antennas based on fractals to cover few kinds of public protection and disaster relief (PPDR) communications was presented. Dedicated antenna forms, necessary for antenna design by $5 \mathrm{G}$ implementation, were enhanced to suit the requirements of specific applications. Employing fractal-shaped antennas have allowed us to accomplish all these actions, which request compact, conformal, and broadband high performance devices. Antennas derived from Koch's curve fractals are studied. In order to implement PPDR communications in $5 \mathrm{G}$ technology, frequency bandwidths of importance have been carefully selected and properly included in the antenna developments under MATLAB environment. Important information necessary for antenna designers, such as 360 degrees directivity at various frequencies, the impedance (resistance and reactance) along the bandwidth of interest, as well as voltage standing wave ratio (VSWR) along the bandwidth of interest for dipole, one-iteration, and two-iteration Koch's curves, respectively, have been obtained. The characteristic of directivity at selected frequencies is also highlighted. In order to maximize antenna parameters, this study has successfully proposed using fractal antennas, objects that use self-similarity property of fractals for optimum operation in several frequency ranges. For the studied antennas, we have obtained the following results regarding the maximum gains in $\mathrm{dBi}$ (which is the unit of the ratio between the gains of the antenna compared to the gain of an isotropic antenna). For the dipole antennas, the gains are $2.73 \mathrm{dBi}$ and $4.76 \mathrm{dBi}$ at $460 \mathrm{MHz}$ and $770 \mathrm{MHz}$, respectively. The gains for one-iteration fractal Koch antenna are $6.91 \mathrm{dBi}$ and $4.51 \mathrm{dBi}$ at $460 \mathrm{MHz}$ and $770 \mathrm{MHz}$, respectively, and finally, for two-iteration fractal Koch antenna, the gains are $4.91 \mathrm{dBi}$ and $3.28 \mathrm{dBi}$ at $460 \mathrm{MHz}$ and $770 \mathrm{MHz}$, respectively. Moreover, the impedance along the bandwidth is approximately 360 Ohms for twoiteration fractal Koch antenna, $180 \mathrm{Ohms}$ for one-iteration fractal Koch antenna, and 140 Ohms for dipole antenna, respectively.
\end{abstract}

\section{Introduction}

Today, the modern society needs more and more information. Apparently, individual customers, also the government, have a fabulous appetite for information repositories. Whether we talk about access to entertainment information or to PPDR (public protection and disaster relief) information, links to information sources are not allowed, from the customer's point of view, to have delays or inability to access them. At this moment, each of us has a smart communications terminal through which we access applications to facilitate our daily activities. 
In this context, being an important part of the transceiver, the antennas must have a suitable design to function accordingly.

The IT\&C environment has begun some time ago to develop programs for most of the actions that a person can take daily. Thus, the possibility of parallelization of activities was born. Moreover, social media applications have developed impressively over the last decade due to the fact that they have allowed people to be interconnected and to share with each other various information. Profile studies have shown that a user of $4 \mathrm{G}$ technology performs double information traffic compared to a user of another type of communication [1].

In the state of the art, a comparison between the results obtained by us in this study and the other authors from the scientific specialized literature is welcomed. Thus, size, shape, and gain are generally better or closer than $[2,3]$, as also highlighted in more depth at a thorough literature review made on this occasion in the next chapters, references cited in text where the exact comparisons are being made. Our values can be found in the results chapter.

Nowadays, mobile operators have developed infrastructures that allow for the sudden development of the IT\&C environment. For this reason, customers are showing today an increasing need for denser networks, greater capacity for high mobility, ubiquitous coverage, low latency, massive number of affiliated devices, and low energy consumption, indices that begin to be decisive when it comes to value for money.

Thus, in order to optimize the production costs, in this document, it is proposed to design a fractal-shaped antenna that can be used for communications in different frequency bands.

Geometric shapes such as Sierpinski's triangle, Koch's curve, or Cantor's set can easily define antennas or antenna networks that facilitate communications across multiple frequency ranges if properly sized.

The study describes the objectives of $5 \mathrm{G}$ communications, the principles of fractal geometry, and how these irregular shapes can help the future of communications.

The work is organized in six chapters. In the introduction, the motivation for developing fractal-shaped antenna in the telecommunication domain is given. The second chapter talks about the emerging need for $5 \mathrm{G}$ technology and all the strict requirements that must be followed in order to allow for a multitude of connected devices to interact in a fast and reliable way. Furthermore, the third chapter introduces the fractal theory and gives few necessary equations. The fourth chapter is devoted to fractals antennas, from description of Koch's curve with governing equations, to enumerating the advantages of fractal-shaped antennas and then moving to fractal antennas design.

In the fifth chapter, the results obtained using our developed MATLAB programs are presented, in specific frequency bandwidth carefully chosen in order to implement PPDR communications in 5G technology. Therefore, the reader is given an overview of important information necessary for antenna designers, such as 360 degrees directivity at various frequencies, the impedance along the bandwidth of interest, as well as voltage standing wave ratio (VSWR) along the bandwidth of interest for dipole, oneiteration, and two-iteration Koch's curves, respectively. The characteristic of directivity at selected frequencies is also highlighted. The study concludes in the sixth chapter.

\section{The Emerging Need for $5 G$}

One desired issue to implement in 5G would be beamforming, the method that should point the end user equipment. Obviously, the cost for $5 \mathrm{G}$ infrastructure would increase because of the huge number of connected devices, fact which is contrary to actual generation of communications, as today, networks use little power to work.

Suddenly, massive MIMO concept became a backup solution to increase the bandwidth but not for a long time because of the high probability of occurrence of interference. So, a good antenna design is needed to avoid this phenomenon.

Microseconds latencies represent one major challenge for $5 \mathrm{G}$. This facility emerged from the rush in which the society is at the moment. Some contemporary processors offer possibilities to achieve this objective, but the laws of physics are the ones that limit the transfers. So, the sender and the receiver should be close enough to accomplish this, and the topology should include a big number of hardware devices.

As for the bandwidths to be used, few intervals are suggested:

(i) $452.5-457.5 \mathrm{MHz}$

(ii) $462.5-467.5 \mathrm{MHz}$

(iii) $753-758 \mathrm{MHz}$

(iv) $788-791 \mathrm{MHz}$

The use of each frequency range implies the emergence of specific behaviors regarding the propagation of electromagnetic waves. Some of the most important phenomena to study are reflection and scattering, free space path loss, diffraction, or material penetration [1].

In order to offer communications for a large number of customers, it is recommended to have intelligent receivers, able to distinguish between signals intended for them, for others or noises. This would become a challenge for DSP or SDR programmers to develop the optimum software.

$5 \mathrm{G}$ technology should be adopted even by the PPDR communications in order to improve their response time in case of an emergency [4].

Combined with fractal design techniques, the new technology should cover almost all the needs of the clients from PPDR area.

\section{Fractals Theory}

In 1977, the Swedish mathematician of French origins, Benoit Mandelbrot (1924-2010), published "Fractal Geometry of Nature," in order to offer a new way of describing the shape of objects such as coastlines and clouds [5]. 
Self-similarity is the most important property of fractals, and it implies that the fractal object must have an identical look at each iteration (most of the natural objects are selfsimilar). Due to the fact that a fractal object cannot be an input for an Euclidean formula, the fractal dimension must be introduced. This notion has a rational value, and it is strictly bigger than topological dimension.

According to Hausdorff, the fractal dimension is proportional to the minimum number of spheres, of given radius required to cover the measured object. Today, cubes are more reliable to use image processing $[6,7]$.

For a curve of length $1, N(s)=1$, cubes of side $s$ are needed. Following this example, for an area $N(s)=1 / s^{2}$, cubes of side $s$ are required, and for a volume, $N(s)=1 / s^{3}$, cubes of side $s$ are required.

Considering $d, s$ from $R$, and $N(s)=f(d)^{*}$ sd, a set of functions, such that $N(s)$ is the number of spheres (balls) of $s$ diameter (cubes of $s$ side), is needed to cover the given set $X$. Then, there is a unique real value $d=D_{H}$, called the Hausdorff dimension of $X$, so that $[7,8]$

$$
d>D_{H} \Rightarrow N(s) \longrightarrow 0 .
$$

Thus, the Hausdorff dimension is described by

$$
N(s) * s D_{H} .
$$

The size of an object describes how it occupies space and how it can be measured (quantitatively). It is expressed in a rational number. We can easily calculate the geometric size of an object [9]. Generally, it is calculated using the Hausdorff dimension DH.

It starts from the formula written above, more precisely

$$
N(s)=f(d)^{*} s^{D_{H}},
$$

where $N(s)$ is the number of cubes (or sphere), $s$ is the length of cube's side (or spheres diameter), and $D_{H}$ is the object's geometric dimension, respectively.

Fractal dimension formula: allow $N(X, s)$ to indicate the minimum series of identical figures (balls, spheres let us say) of radius $s$, necessary to include in the set $X$. Then, the theoretical fractal dimension is exactly given as

$$
d(X)=\lim _{s \longrightarrow 0} \sup \frac{\log N(X, s)}{\log (1 / s)} .
$$

Observation 1: the coherent definition of fractal dimension we demonstrate here would essentially be proven if the superior limit in (4) could be superseded by a straightforward limit. No, this is not an extravagant exigency, and the "lim sup" solicitation is necessary, as there are simple sets for which the limit as $s \longrightarrow 0$ is nonexistent [8].

In concluding this topic, we specify that there are some very good methods for fast calculation of the fractal dimension, among which we list the one called the boxcounting method.

Observation 2: on the engineering expectation side, the practical box-counting dimension could replace the

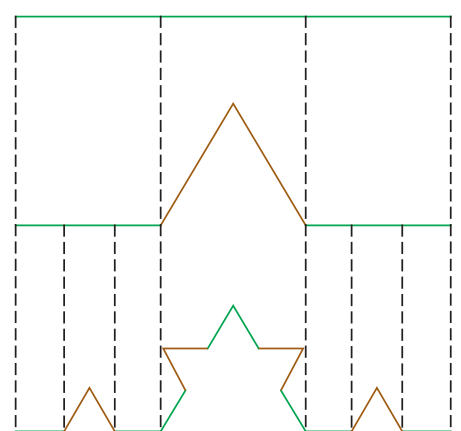

FIgURE 1: Koch curve construction based on Koch generator, orders 0,1 , and 2 .

theoretical Hausdorff-Besicovitch dimension, being given by the box-counting method, facile to calculate. However, the aspect of fractal dimension calculus being nonetheless beyond the purpose of the current study, we will stop here with the details related to this topic. Fractals used for antenna design are artificial ones like Minkowski's loop, Koch's curve, Sierpinski's carpet, and Sierpinski's gasket

\section{Fractals Antennas}

As we can affirm now, one of the advantages of fractal object is that it has an infinite measure of length, fitting in a finite measure of volume. Like it is known, radiation frequency is distinct for each individual electromagnetic transmitter and depends on electrical length of geometric structure. That is why, it was considered that utilizing the property of fractal geometry, we may increase the electrical length of an antenna (in direct connection with the wavelength, respectively), maintaining the identical antenna volume. We rely on mathematical observation at our disposal, namely, that we have many possibilities available to attempt as a design of same fractal antenna [10].

4.1. Fractal Geometry. Certain fractals portray replica of the entire structure at reduced scales. This property is mathematically named self-similarity, and it is helpful to project multiband antennas. Koch's snowflake is one of the particularly familiar self-similar fractals. Some other fractals fill the space, as is the case of space-packing curves, which makes them attractive in the design of little antennas. Koch's curve is one example of a space-packing curve, which has been utilized to build little antennas [11].

4.1.1. Koch's Curve. The initiator or, more precisely, the initial curve is a straight line. Specifically, in this case, the law of transformation requires that the straight line be divided into three equal parts that the central part be removed and in its place, an equilateral triangle without a base be placed (Figure 1).

At each iteration, each independent right is considered, and the transformation law is applied to it. In this case, the 4 segments each become a "new" initiator, and the support of 4 

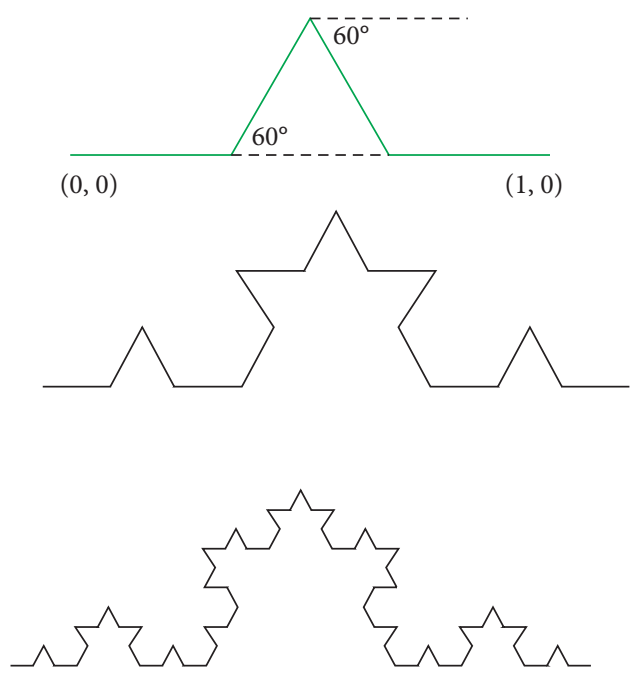

FIGURE 2: Koch curve construction based on the generator, orders 1 and 2 and length $=4 / 3$.

"images" shrunk and is placed according to the same rule. Let us not forget that our mind must take the essence of the process and continue it to infinity because only after infinity of many steps, we obtain what is called the Koch fractal. This curve is of infinite length and has its own dimension between 1 and 2. It is a "strange" geometric object for the thinking of a person, unused to work with abstract things. It is a continuous curve, indivisible at no point, which exceeds the "nature" of a line, but does not reach the quality of being surface [11].

The proper fractal dimension: the characteristic of this Koch curve is fractal dimension: $\mathrm{d} f=\ln (4) / \ln (3)=1.26185 \ldots$.

A more concrete explanation is that to construct this curve, one starts by drawing a straight line (the blue segment in the figure). Then, divide this segment into three equal parts, and the middle segment is replaced by the two sides of an equilateral triangle of the same length as the length of the segment that is removed (the two red segments in the middle of the figure) [9]. Now repeat, take each of the four resulting segments, divide them into 3 equal parts, and replace each of the middle segments in two sides of an equilateral triangle (the red segments at the bottom of the figure).

In Figure 1, the Koch curve construction based on a specific generator is presented, namely, orders 0,1 , and 2 .

The Koch curve is the limit of a curve obtained by applying this construction an infinite number of times. As proof, this construction even produces a "limit" that is the current curve, for example, the continuous image of a compact interval.

The first iteration for the Koch curve consists the fact that 4 copies of the original right segment are taken, each multiplied by $r=1 / 3$. Two segments must be rotated by $60^{\circ}$, one clockwise and one in reverse.

In Figure 2, the Koch curve construction, based on a specific generator, is presented. Obviously, the length obtained is $4 / 3$.
Niels Fabian Helge von Koch (1870-1924) constructed this curve in 1904 as an example of a nondifferentiable curve, which is a continuous curve that has no tangents at any point. Karl Weierstrass demonstrated the first existence of such a curve in 1872. The length of the intermediate curve at the $n^{\text {th }}$ iteration of the construction is $(4 / 3) n$, where $n=0$ denotes the original length of the right segment. However, the length of the Koch curve is infinite. Moreover, the length of the curve between any 2 points of the curve is also infinite, with a copy of the Koch curve between any 2 of its points $[12,13]$.

4.2. Advantages of Fractal-Shaped Antennas. The telecommunications branch considers the antennas as a separate element of radio communications and therefore needs special attention not only in the design phase but also in the maintenance phase.

As the world becomes more and more dependent on cellular devices, there has been an increasing demand in antennas that are compact, conformal, and broadband [10]. A popular method of achieving these characteristics in an antenna is by exploiting the property of fractals.

In order to maximize antenna's length, this study suggests using fractal antennas, objects that use self-similarity property of fractals for optimum operation in several frequency ranges.

Among the advantages of fractal-shaped antennas, we can mention the following:

(i) Very compact emplacement (a wire can be bended as a fractal, and so at the end of the process, on the same surface will fit a larger length of wire)

(ii) Higher input resistance because of increase in length or perimeter [4]

(iii) Low side-lobe levels and wide bandwidth

(iv) Capable to implement beam-forming techniques due to fractals recursivity [10]

According to a public document from National Authority for Management and Regulation in Communications of Romania (ANCOM) (page 49) [14], PPDR communications would use few bandwidths to specific links. So, why do not the systems use only one antenna for all bandwidths? This can be the possibility to develop a self-reconfigurable system.

4.3. Fractal Antennas Design. The support of fractals geometry in antenna design engineering has led to a novel recently acquainted domain like fractal-electrodynamics, which has greatly influenced antenna theory. Traditional antenna design has been based on Euclidean geometries, but novel designs have come from modern fractal antenna engineering.

Fractal antennas get the benefit of geometries that cannot be acquired by classical Euclidean developments to provide accurately what consumers want. 


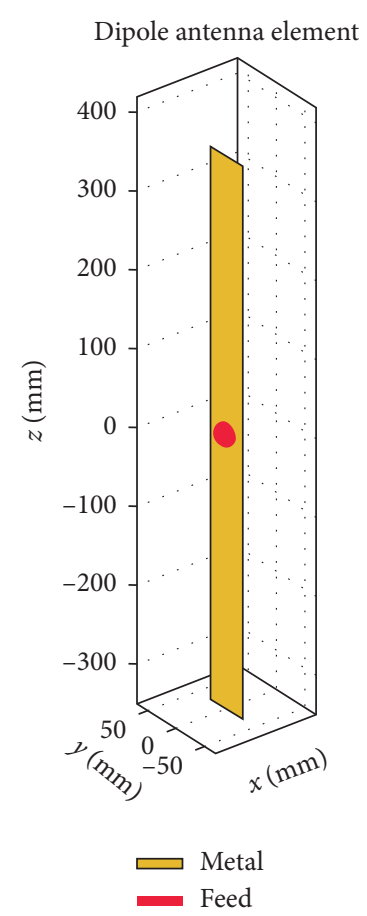

Figure 3: Dipole antenna.

First of the significant profit of fractal antenna is that we can obtain more than one single resonant band. As we said before, the fractal concept can be used to reduce antenna dimensions, such as in the case of the Koch dipole, Koch monopole, Koch loop, and Minkowski loop, for example.

The relationship that describes the radiated power for a multielement antenna is the following [13]:

$$
E \cdot E^{*} \sim\left(\sum_{n=1}^{N} A_{n} e^{i \phi_{n}}\right) \cdot\left(\sum_{m=1}^{N} A_{n} e^{i \phi_{m}}\right)^{*}=\sum_{n, m}\left(A_{n} A_{m}^{*}\right) e^{i\left(\phi_{n}-\phi_{m}\right)},
$$

where $A_{n}$ defines the strength and orientation for each element, and $\phi_{n}$ has a connection with fractal's elements spatial distribution. We mention that every one of the elements concurs at the calculus of the entire radiated power density at a time-determined moment, through a vectorial amplitude and known phase, evidently.

A difference between antenna arrays and fractal antenna is that the order of classic antennas makes them be resonant just along one bandwidth, and fractal's disorder (made by fractal's elements) makes fractal-shaped antenna be resonant along many bandwidths (iteration 0 can be viewed as a classic antenna, and starting with a new iteration, a new bandwidth can be obtained).

Several applications can be developed based on fractalshaped antennas due to their compact size and multiband resonance. Applications such as radars, personal cell phones, the ones from UWB branch, or 5G PPDR devices can benefit such an element which can have as a starting point any dedicated antenna (log-periodic, monopole, dipole, and patch).

\section{Results}

In order to implement PPDR communications in 5G technology, few bandwidths were suggested by ANCOM in Romania [14]: 452.5-457.5 MHz, $462.5-467.5 \mathrm{MHz}$, 753-758 MHz, and 788-791 MHz.

5.1. Antennas Derived from Koch's Curve Fractals. From this point of view, we designed in MATLAB R2018b a center-fed fractal-shaped antenna (Koch's curve format) to observe how well does this kind of solution fits in a $5 \mathrm{G}$ communication in comparison with a dipole.

By default, MATLAB generates the dipole with a length of one meter each side and 0.1 meters width. By adjusting the dimensions according to the desired bandwidths, we obtain a length of 0.75 meters each side and a width of 0.0583 meters. After making the first iteration, we observe that the antenna should be fatten in order to obtain a wider bandwidth [13]. Thus, the length obtained is 0.7 meters for one side and the width is 0.07 meters. Achieving the second iteration, it can be observed that no fatten is needed. So, the length would be the same, 0.7 meters for each side, and the width would also remain 0.0583 meters.

Figure 3 presents the dipole antenna's element, as simulated.

In Figures 4-6, the 360 degrees directivity (for dipole, one-iteration Koch's curve and two-iteration Koch's curve), at frequency value of $460 \mathrm{MHz}$ and frequency value of $770 \mathrm{MHz}$, are shown in detail.

The resonant frequency can be determined using fractal dimension by dividing $c\left(3 \cdot 10^{8} \mathrm{~m} / \mathrm{s}\right)$ to 4 multiplied by the effective length of fractal [10].

Figures 7 and 8 display the three-dimensional radiation pattern and phase of the dipole at $460 \mathrm{MHz}$ and $770 \mathrm{MHz}$, respectively, in a color-coded fashion.

It can be observed in Figure 9 that the impedance is almost 50 Ohms along the bandwidths of interest [15].

In Figure 10, the fractal Koch antenna (iteration 1) is represented, while further on, Figure 11 depicts the Fractal Koch antenna (iteration 2).

In Figure 5, one-iteration fractal Koch antenna 360 degrees directivity is graphically represented for the two frequencies of interest, 460 and $770 \mathrm{MHz}$, respectively.

Figures 12 and 13 show the color-coded three-dimensional representation of one-iteration fractal Koch antenna radiation pattern and phase at $460 \mathrm{MHz}$ and $770 \mathrm{MHz}$, respectively [16, 17].

It can be observed in Figure 14 that the impedance has zero imaginary component, so the antenna is resonant along the bandwidths of interest.

Figures 15 and 16 show the color-coded three-dimensional representation of two iterations: fractal Koch antenna radiation pattern and phase at $460 \mathrm{MHz}$ and $770 \mathrm{MHz}$, respectively.

Figure 17 shows that along two of the bandwidths of interest, the antenna might not be resonant, but it still remains suitable for the two others. 


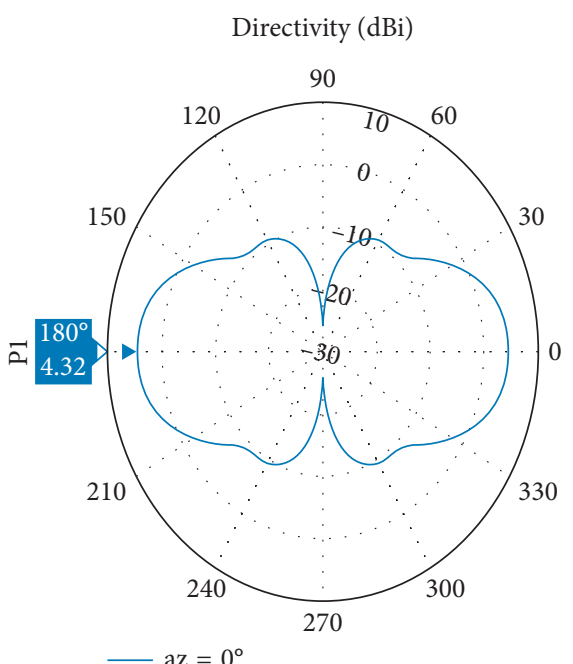

$-\mathrm{az}=0^{\circ}$

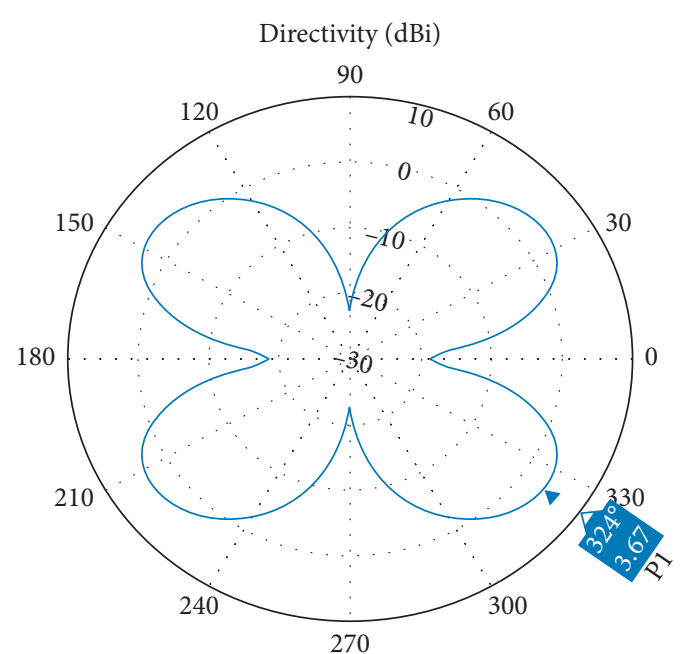

$-\mathrm{az}=0^{\circ}$

(a)

(b)

Figure 4: Dipole's 360 degrees directivity (more precisely, for dipole at $460 \mathrm{MHz}$ left and right at $770 \mathrm{MHz}$ ).

Directivity (dBi)

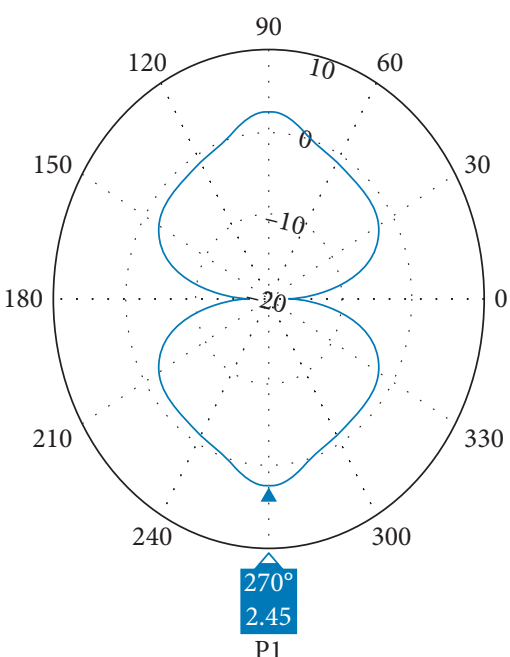

$-\mathrm{az}=0^{\circ}$

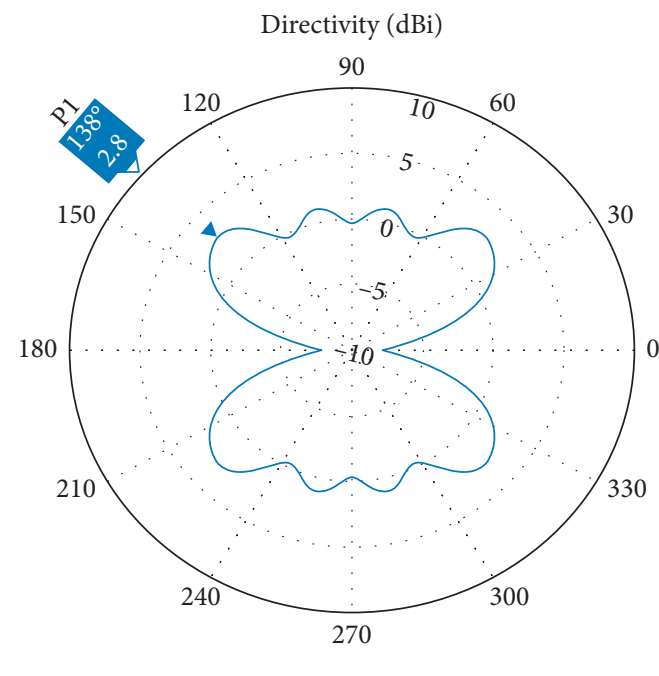

$-\mathrm{az}=0^{\circ}$

(a)

(b)

FIGURE 5: One-iteration fractal Koch antenna 360 degrees directivity (more precisely, one-iteration Koch's curve at $460 \mathrm{MHz}$ left and right at $770 \mathrm{MHz}$ )

In Figures 9, 14, and 17, the impedance, measured in Ohms (on the $y$-axis), is graphically represented along the band of interest versus frequency, measured in $\mathrm{GHz}$ [17], for the antenna with dipole, with one-iteration, and with two-iteration of Koch's curve. The graphics are marked with two distinct colors, resistance (blue) and reactance (red), to distinguish them.

Values for impedance, from Figures 9, 14, and 17, show that the dipole and the one-iteration Koch's curve antennas are suitable for using in the desired bandwidths, and the two- iteration Koch's curve may work poorly in the first part of the chosen spectrum, but it will certainly work properly in the second half.

The VSWR, Figure 18, it can be observed that most of the power to be delivered to the antennas is actually radiated by the antennas. For the first couple of bandwidths, the dipole and the two-iteration fractal are the most suitable, whereas for the second couple of bandwidths, the antenna from Figure 10 is better. 


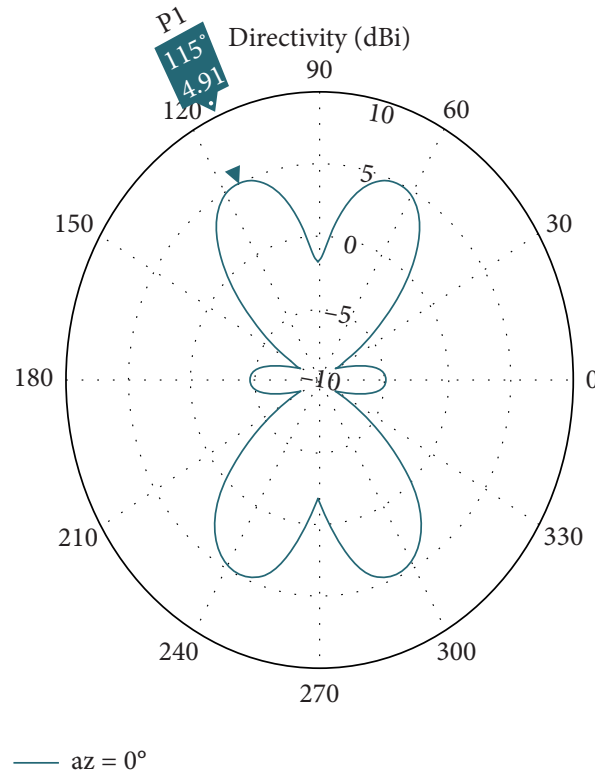

(a)

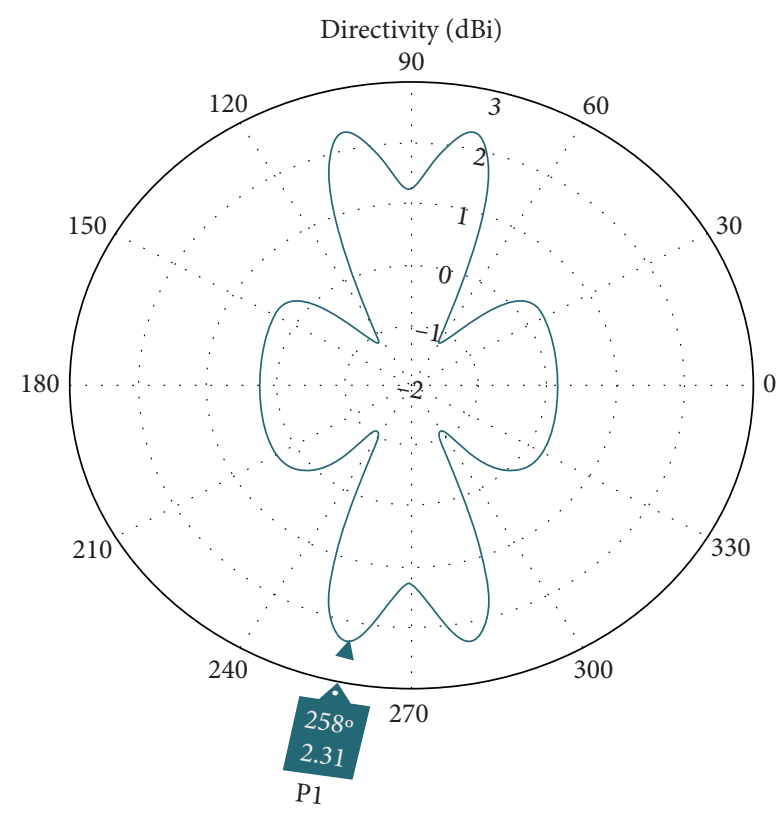

(b)

FIGURE 6: Two-iteration fractal Koch antenna 360 degrees directivity (more precisely, two-iteration Koch's curve at $460 \mathrm{MHz}$ left and right at $770 \mathrm{MHz}$ ).

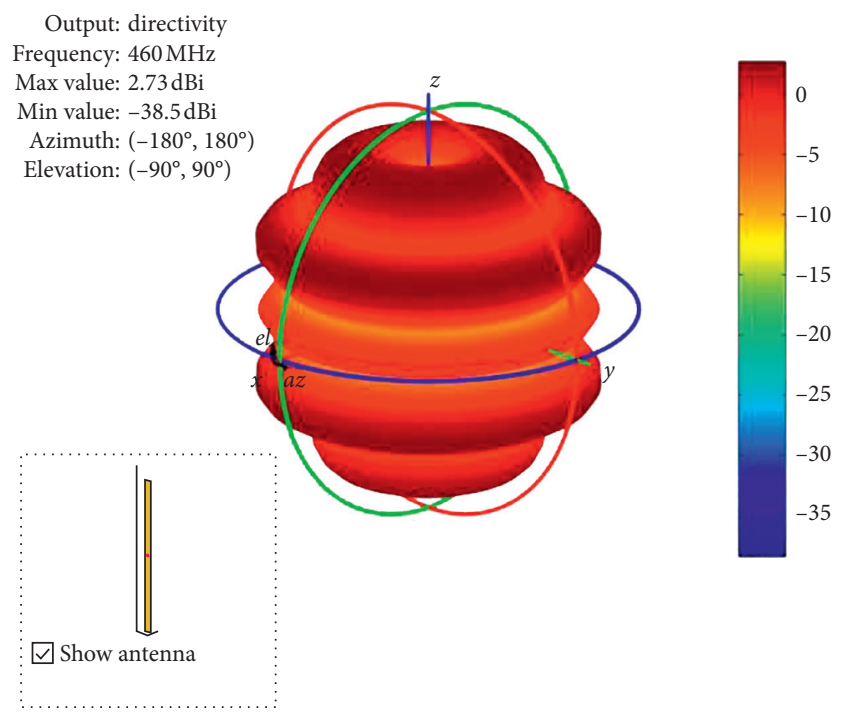

Figure 7: Dipole's radiation pattern and phase at $460 \mathrm{MHz}$.

In Figure 18, the magnitude, measured in $\mathrm{dB}$ (on the $y$ axis), is graphically represented along the band of interest versus frequency, measured in $\mathrm{MHz}$ for the antenna with dipole (iteration 0), with one-iteration, and with two-iteration of Koch's curve. The graphics are marked with three distinct colors for dipole (blue), one-iteration (brown), and two-iteration (olive) to distinguish them.

The VSWR values, from Figure 18, show that most of the power to be delivered to the antennas is actually radiated by the antennas. For the first couple of bandwidths, the dipole and the two-iteration fractal are the most suitable, whereas for the second couple of bandwidths, the resulting antenna is much better $[2,18,19]$.

In Figure 19, the return loss for dipole and Koch's curve antennas have been evaluated, where the magnitude, measured in $\mathrm{dB}$ (on the $y$-axis), is graphically represented versus frequency, measured in $\mathrm{MHz}$ [20]. The graphics are marked with three distinct colors for dipole (blue), one-iteration (brown), and two-iteration (olive) to distinguish them.

In Figure 20, a comparison between dipole and Koch's curves antennas (one-iteration and two-iteration) is shown. Also, the graphics are marked with three distinct colors, blue, brown, and olive for dipole, one-iteration, and twoiteration, respectively.

We would like at this point to give a summary of the quantitative results we have obtained in our study. By performing the three-dimensional physical simulations of the artefacts developed, we have obtained the following numerical values for the directivity with maximum values of $2.73 \mathrm{dBi}$ and $4.76 \mathrm{dBi}$ for the dipole at $460 \mathrm{MHz}$ and $770 \mathrm{MHz}$, respectively. At the same frequencies, the antenna radiation patterns revealed at $460 \mathrm{MHz}$ maximum directivity values of $6.91 \mathrm{dBi}$ for one-iteration fractal Koch antenna and $4.91 \mathrm{dBi}$ for two-iteration fractal Koch antenna, while at $770 \mathrm{MHz}$, those values were $4.51 \mathrm{dBi}$ for one-iteration fractal Koch antennas and $3.28 \mathrm{dBi}$ for two-iteration fractal Koch antenna, respectively. Therefore, we can observe that the directivity drops by $29 \%$ for one-iteration antenna at $460 \mathrm{MHz}$ and by approximately $27 \%$ at the higher frequency 
Output: directivity Frequency: $770 \mathrm{MHz}$ Max value: $4.76 \mathrm{dBi}$ Min value: $-44.9 \mathrm{dBi}$ Azimuth: $\left(-180^{\circ}, 180^{\circ}\right)$ Elevation: $\left(-90^{\circ}, 90^{\circ}\right)$

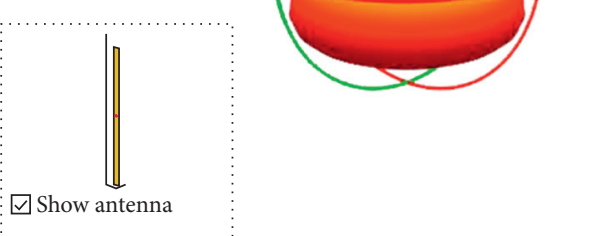

Figure 8: Dipole's radiation pattern and phase at $770 \mathrm{MHz}$.

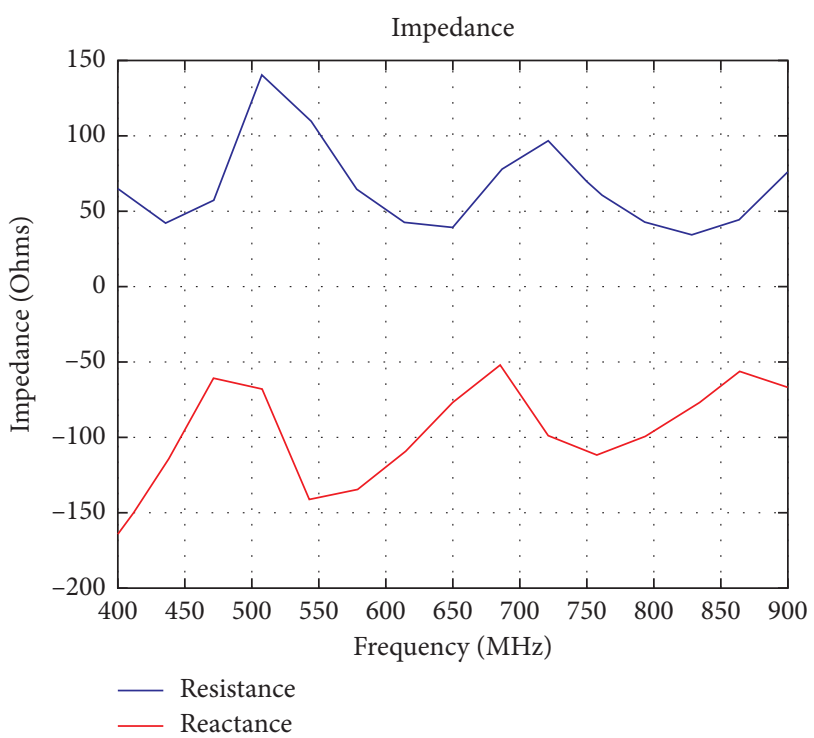

FIGURE 9: Dipole's impedance along the bandwidth.

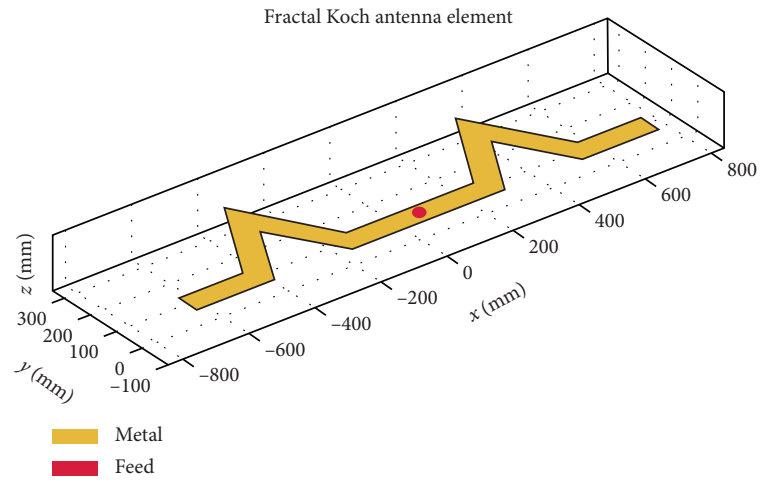

Figure 10: Fractal Koch antenna (iteration 1).

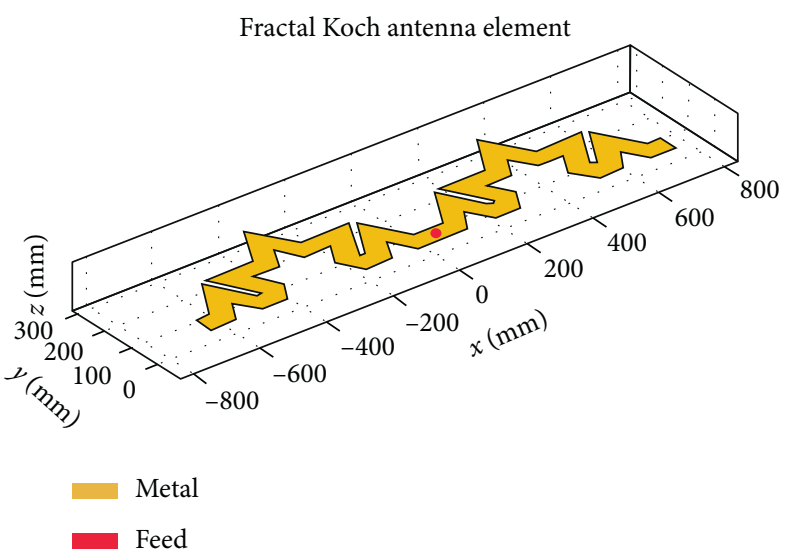

FIgURE 11: Fractal Koch antenna (iteration 2).

Output: directivity Frequency: $460 \mathrm{MHz}$ Max value: $6.91 \mathrm{dBi}$ Min value: $-24.7 \mathrm{dBi}$ Azimuth: $\left(-180^{\circ}, 180^{\circ}\right)$ Elevation: $\left(-90^{\circ}, 90^{\circ}\right)$
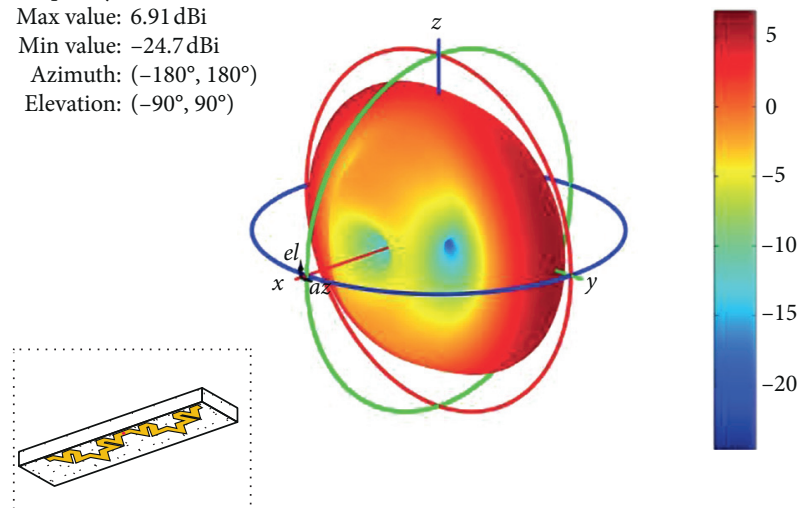

Dhow antenna

Figure 12: One-iteration fractal Koch antenna radiation pattern and phase at $460 \mathrm{MHz}$.

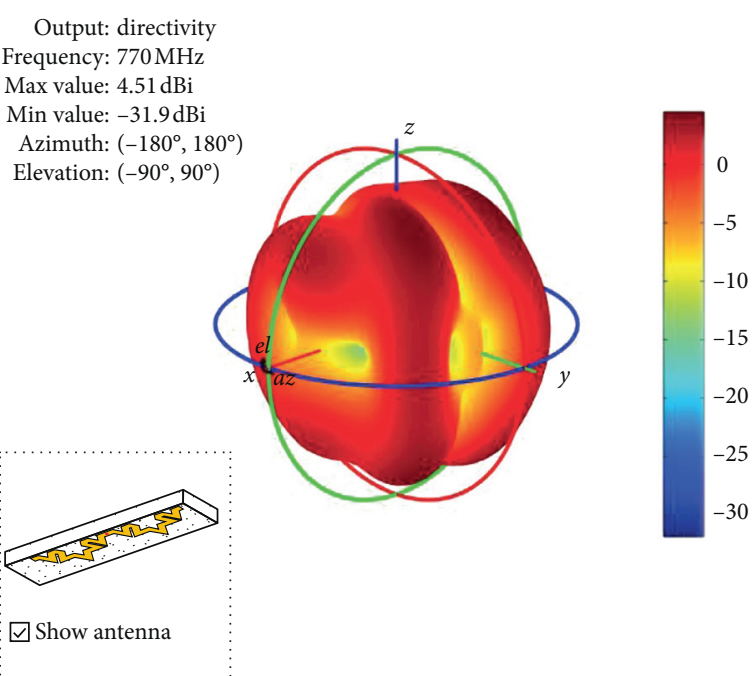

Figure 13: One-iteration fractal Koch antenna radiation pattern and phase at $770 \mathrm{MHz}$. 


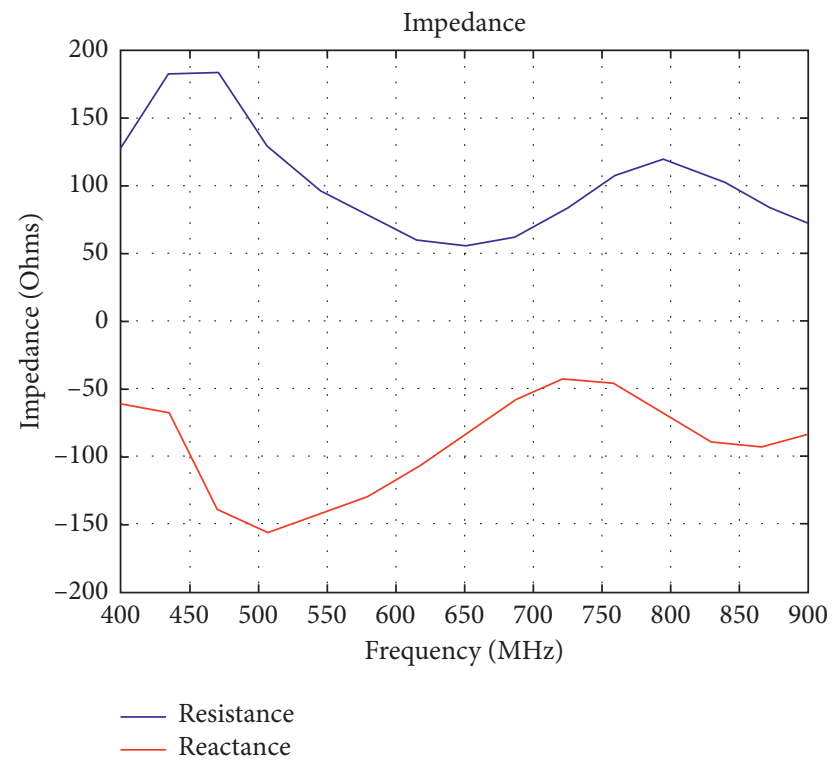

FIGURE 14: One-iteration fractal Koch antenna impedance along the bandwidth.

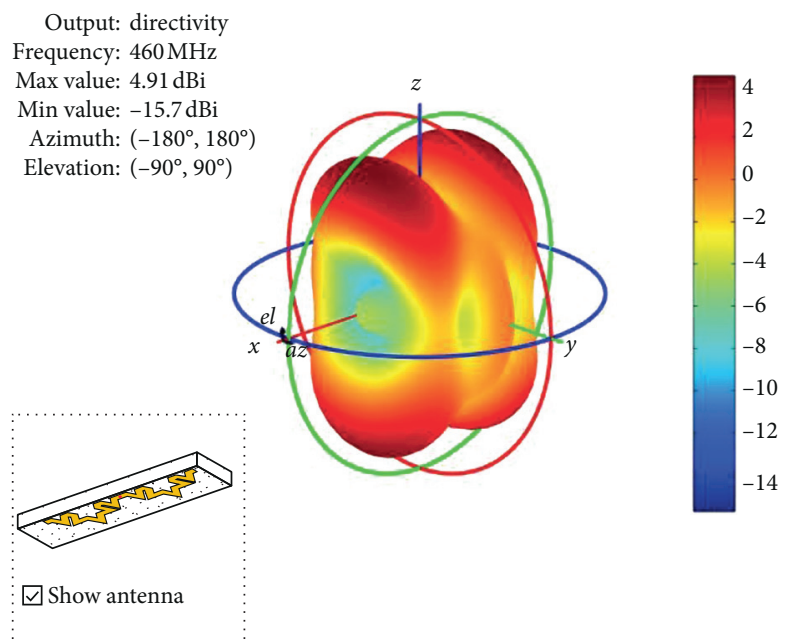

Figure 15: Two-iteration fractal Koch antenna radiation pattern and phase at $460 \mathrm{MHz}$.

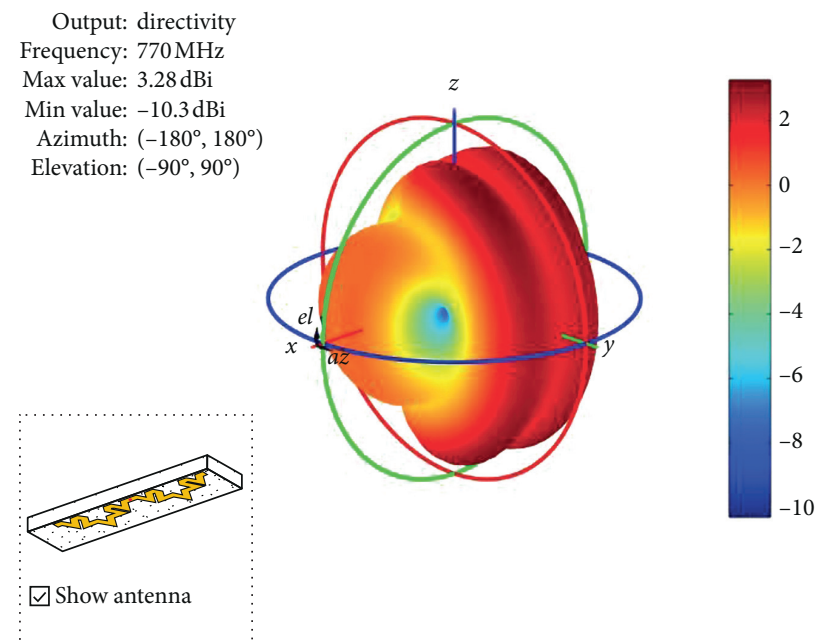

FIgURE 16: Two-iteration fractal Koch antenna radiation pattern and phase at $770 \mathrm{MHz}$. 


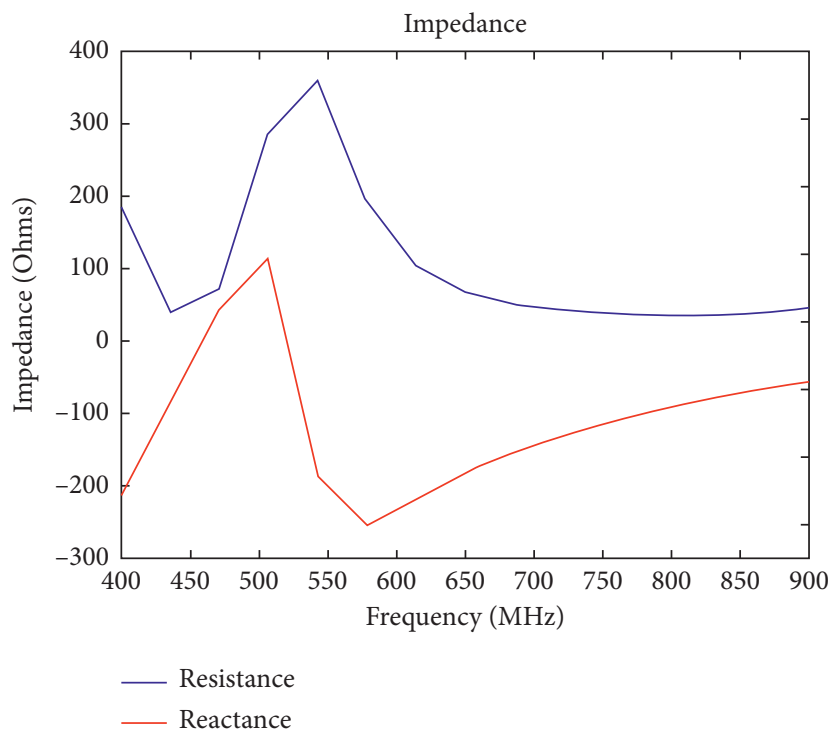

FIgURE 17: Two-iteration fractal Koch antenna impedance along the bandwidth.

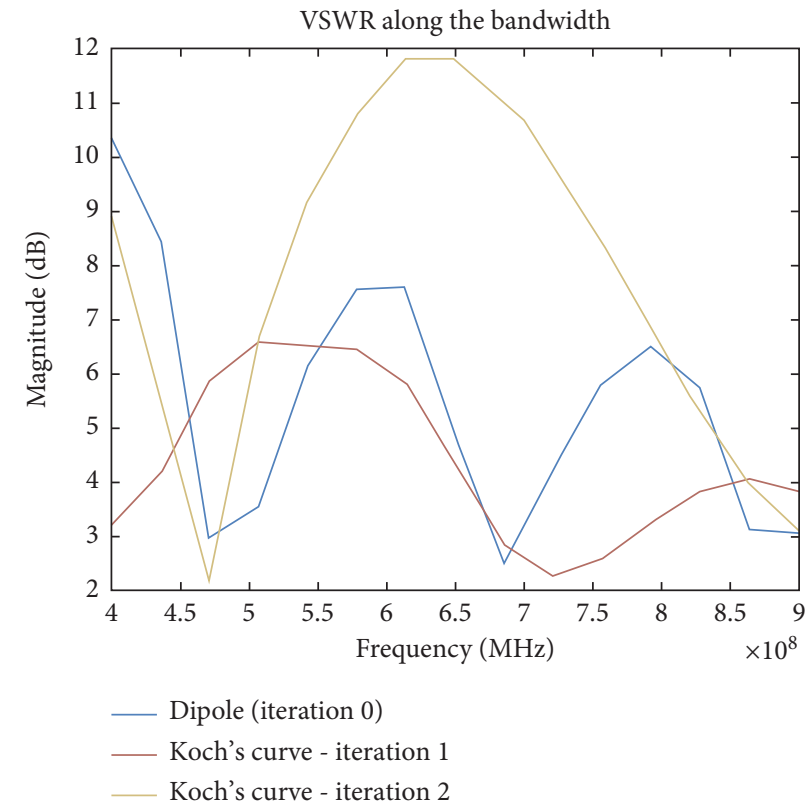

FIgURE 18: VSWR for dipole and Koch's curve antennas.

of $770 \mathrm{MHz}$. Moreover, both one-iteration and two-iteration fractal Koch antennas exhibit higher maximum directivities than dipole antennas at both frequencies studied.

The VSWR investigation produced the highest value of approximately $12 \mathrm{~dB}$ at $625 \mathrm{MHz}$ for two-iteration fractal Koch antenna. Moreover, maximum return loss magnitudes of approximately $8.8 \mathrm{~dB}$ for two-iteration Koch antenna and of $6 \mathrm{~dB}$ for dipole antenna are obtained at $460 \mathrm{MHz}$. Finally, the impedance along the bandwidth is approximately 360 Ohms for two-iteration fractal Koch antenna, $180 \mathrm{Ohms}$ for one-iteration fractal Koch antenna, and $140 \mathrm{Ohms}$ for dipole antenna, respectively. 


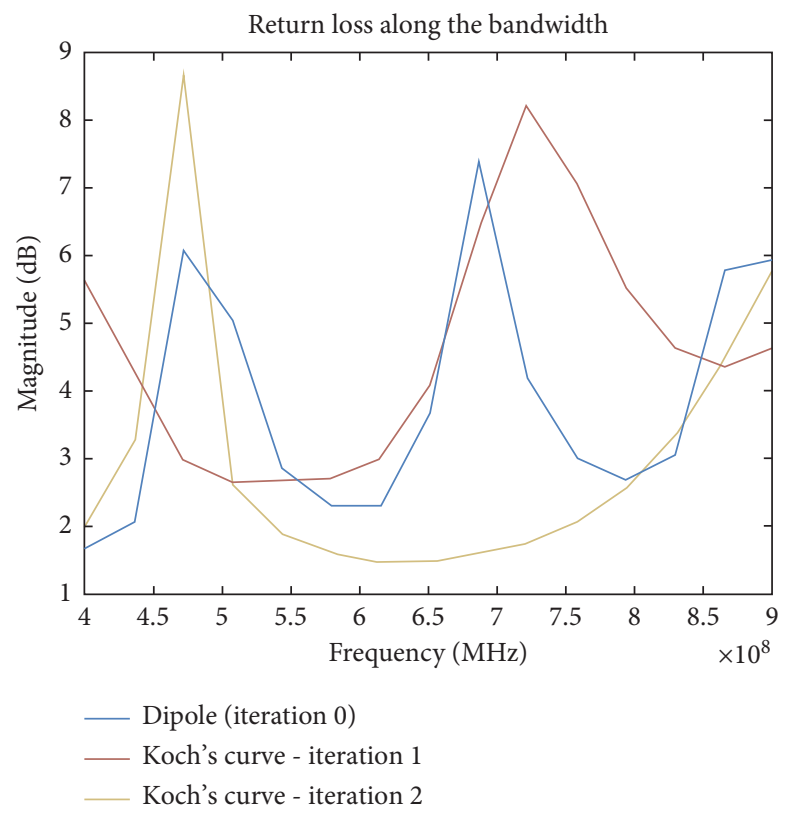

Figure 19: Return loss for dipole and Koch's curve antennas.

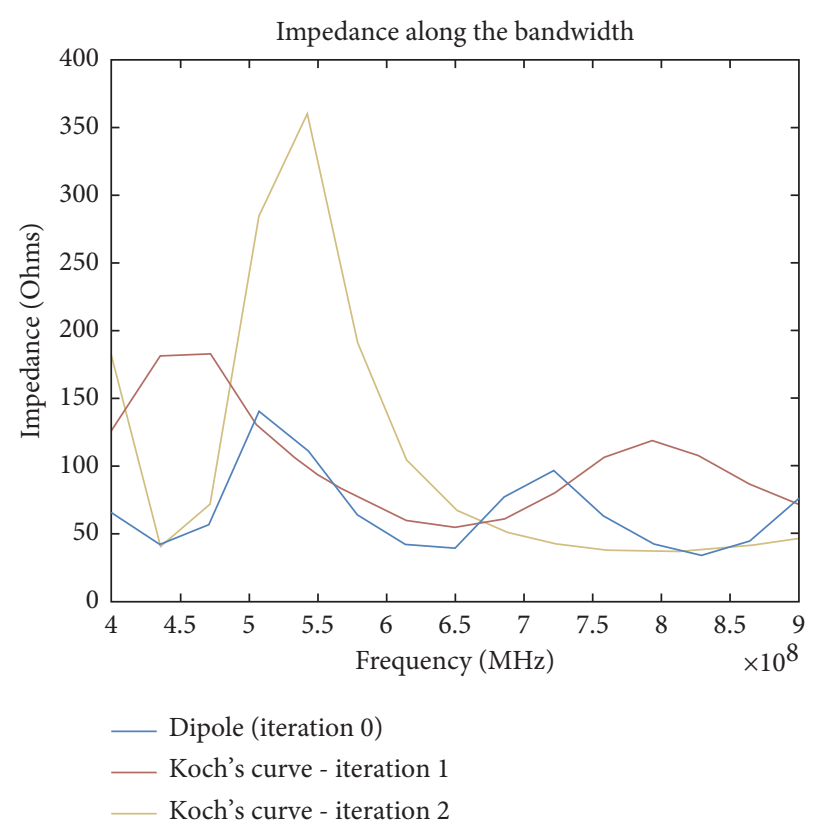

Figure 20: Comparison between dipole and Koch's curves antennas.

\section{Conclusions}

Dedicated wideband antennas can be used as starting point for fractal-shaped antennas to obtain new advantages such as compact size, multiband resonance, and providing more communication links to a single device.

One problem for the emergent technology, 5G, is the antenna because it has to transfer a huge quantity of information without filtering a little slice. This can be very challenging when a large number of users and protocols to follow are taken into account.
Only one antenna can cover technologies such as 2G, 3G, $4 \mathrm{G}$, ZigBee, WLAN, and WiMax if properly designed, and it would be a shame to forget these kinds of networks when $5 \mathrm{G}$ would appear on the market.

Because of the need for speed, in order to improve the response times, the PPDR agencies will adopt the 5G technology as soon as possible.

In this study, two ways of using fractal-based antennas to cover few kinds of PPDR communications are presented.

The fractal shape suggested is a Koch's curve fractalshaped antenna, which was based on a dipole. This kind of 
antenna covers frequencies between $350 \mathrm{MHz}$ and $800 \mathrm{MHz}$ with few limitations.

All results were obtained using MATLAB R2018b software development. For the studied antennas, we have obtained the following results regarding the maximum gains in $\mathrm{dBi}$ (which is the unit of the ratio between the gains of the antenna compared to the gain of an isotropic antenna). For the dipole antennas, the gains are $2.73 \mathrm{dBi}$ and $4.76 \mathrm{dBi}$ at $460 \mathrm{MHz}$ and $770 \mathrm{MHz}$, respectively. The gains for one-iteration fractal Koch antenna are $6.91 \mathrm{dBi}$ and $4.51 \mathrm{dBi}$ at $460 \mathrm{MHz}$ and $770 \mathrm{MHz}$, respectively, and finally, for twoiteration fractal Koch antenna, the gains are $4.91 \mathrm{dBi}$ and $3.28 \mathrm{dBi}$ at $460 \mathrm{MHz}$ and $770 \mathrm{MHz}$, respectively. Moreover, the impedance along the bandwidth is approximately 360 Ohms for two-iteration fractal Koch antenna, $180 \mathrm{Ohms}$ for one-iteration fractal Koch antenna, and $140 \mathrm{Ohms}$ for dipole antenna, respectively.

Signal intelligence, electronic warfare, or tactical communications can be one of those applications because properties such as wideband frequency range or compact size are desired for the ones who develop solutions for this branch.

Therefore, objectives raised for antenna design by $5 \mathrm{G}$ implementation can be accomplished by enhancing dedicated antenna forms. These actions can be done using fractal-shaped antennas. Much more, in the future, we intend to use an original two or three-iteration binary bionic fractal tree as the pattern in antenna to design 5 th generation (5G) antennas, derived from the rules of nominal microstrip monopole antenna but together with the resonant connection device [21], integrated in the benefit of familiar fractal Koch geometry.

\section{Data Availability}

The data used to support the findings of this study cannot be accessed due to commercial confidentiality.

\section{Conflicts of Interest}

The authors declare that they have no conflicts of interest.

\section{References}

[1] M. V. Nichita, P. Ciotirnae, R. L. Luca, and V. N. Petrescu, "5G propagation: current solutions and future proposals," in Proceedings of the 2016 12th IEEE International Symposium on Electronics and Telecommunications (ISETC), pp. 47-50, Timisoara, Romania, October 2016.

[2] G. Khanna and N. Sharma, "Fractal antenna geometries: a review," International Journal of Computer Applications, vol. 153, no. 7, pp. 29-32, 2016.

[3] N. Mohamed Mohamed, E. Antonino-Daviu, M. CabedoFabrés, and M. Ferrando-Bataller, "A novel low-profile highgain UHF antenna using high-impedance surfaces," IEEE Antennas And Wireless Propagation Letters, vol. 14, pp. 1014-1017, 2015.

[4] https://www.mathworks.com/help/antenna/fractal-antennas. html, website accessed on 24th of February 2020.

[5] B. Mandelbrot, Fractal Geometry of Nature, pp. 25-57, Freeman, New York, NY, USA, 1983.
[6] M. V. Nichita and V. P. Paun, "Fractal analysis in complex arterial network of pulmonary X-rays images," University Politehnica of Bucharest Scientific Bulletin, Series A-Applied Mathematics and Physics, vol. 80, no. 2, pp. 325-339, 2018.

[7] C. A. Rogers, Hausdorff Measures, Cambridge University Press, Oxford, UK, 1970.

[8] M. V. Nichita, M. A. Paun, V. A. Paun, and V. P. Paun, "Fractal analysis of brain glial cells. Fractal dimension and lacunarity," University Politehnica of Bucharest Scientific Bulletin, Series A-Applied Mathematics and Physics, vol. 81, no. 1, pp. 273-284, 2019.

[9] D. Bordescu, M. A. Paun, V. A. Paun, and V. P. Paun, "Fractal analysis of neuroimagistic. Lacunarity degree, a precious indicator in the detection of Alzheimer's disease," University POLITEHNICA of Bucharest Scientific Bulletin, Series A-Applied Mathematics and Physics, vol. 80, no. 4, pp. 309-320, 2018.

[10] A. Kiani, S. T. Karim, and S. B. Solak, Fractal Antennas, McGill University, Montreal, Canada, 2007.

[11] Y. K. Choukiker and S. K. Behera, "Wideband frequency reconfigurable Koch snowflake fractal antenna," IET Microwaves, Antennas \& Propagation, vol. 11, no. 2, pp. 203-208, 2017.

[12] H. Rajabloo, V. Amiri Kooshki, and H. Oraizi, "Compact microstrip fractal Koch slot antenna with ELC coupling load for triple band application," AEU - International Journal of Electronics and Communications, vol. 73, pp. 144-149, 2017.

[13] N. S. M. Yaziz, M. K. A. Rahim, F. Zubir, N. S. Nadzir, R. Dewan, and H. A. Majid, "The improvement of first iteration log periodic fractal Koch antenna with slot implementation," International Journal of Electrical and Computer Engineering (IJECE), vol. 8, no. 4, pp. 2564-2570, 2018.

[14] https://www.ancom.ro/uploads/links_files, website accessed on 14th of March 2020..

[15] Z. Borsos, V.-P. Paun, I. C. Botez, C.-M. Stoica, P. Vizureanu, and M. Agop, "Structural conductivity of carbon nanotubes," Revista de Chimie, vol. 59, no. 10, pp. 1169-1171, 2008.

[16] J. Anguera, A. Andújar, J. Jayasinghe et al., "Fractal antennas: an historical perspective", review, fractal fract," $M D P I$, vol. 4, no. 1, pp. 1-26, 2020.

[17] R. Tyagi and S. Kohli, "Design and development of square fractal antenna for wireless applications," International Journal of Advanced Research in Computer Science and Software Engineering, vol. 5, no. 5, 2015.

[18] P. Srivastava and O. P. Singh, "A review paper on fractal antenna and their geometries," in Proceedings of the Advances in Electrical \& Information Communication Technology, AEICT, 2015, Kanpur, India, April 2015.

[19] M.-A. Paun and V.-A. Paun, "High-frequency 3-D model for the study of antennas in cochlear implants," IEEE Transactions on Components, Packaging and Manufacturing Technology, vol. 8, no. 7, pp. 1135-1140, 2018.

[20] B. Lazar, A. Sterian, S. Pusca, V. Paun, C. Toma, and C. Morarescu, "Simulating delayed pulses in organic materials," in Proceedings of the Conference: International Conference on Computational Science and its Applications (ICCSA 2006), Computational Science and its Applications - ICCSA 2006, vol. 3980, pp. 779-784PT 1 Book Series: Lecture Notes in Computer Science, Glasgow, UK, May 2006.

[21] X. Ran, Z. Yu, T. Xie, Y. Li, X. Wang, and P. Huang, "A novel dual-band binary branch fractal bionic antenna for mobile terminals," International Journal of Antennas and Propagation, vol. 2020, Article ID 6109093, 9 pages, 2020. 